\title{
Eficiencia del transporte urbano de pasajeros en la localidad de Bahía Blanca, Argentina 2007-2014*
}

\author{
Recibido: 05 de mayo de 2016 • Aprobado: 08 de septiembre de 2016 \\ DOI: DOI: 10.22395/seec.v19n41a2 \\ Valentina Viego* \\ Carolina Volontét**
}

\section{RESUMEN}

El objetivo de este artículo es evaluar la eficiencia en la prestación del servicio de transporte urbano por colectivo en la ciudad de Bahía Blanca, Argentina. Las estimaciones se realizaron mediante la aplicación del método de frontera estocástica a una función de producción con datos de panel para el período 2007-2014. Los resultados indican que el nivel medio de eficiencia no varió significativamente durante el período analizado. Asimismo, las transferencias monetarias y la concentración de líneas permitieron mantener la rentabilidad empresarial sin que ello se tradujera en ganancias de eficiencia.

\section{PALABRAS CLAVE}

Movilidad urbana, transporte público de pasajeros, frontera estocástica, Bahía Blanca, Argentina.

\section{CLASIFICACIÓN JEL}

R41, R48

\section{CONTENIDO}

Introducción; 1. Metodología y datos; 2. Resultados; 3. Discusión; 3. Conclusiones; Referencias.

Este artículo de investigación es producto del proyecto titulado "Movilidad intraurbana en la localidad de Bahía Blanca: modalidades y factores condicionantes", el cual fue financiado con fondos de la Secretaria de Ciencia y Tecnología de la Universidad Nacional del Sur. Período de ejecución: 2015-2016.

** Licenciada en Economía, Universidad Nacional del Sur, Bahía Blanca, Argentina. Doctora en Economía Universidad Nacional del Sur, Bahía Blanca, Argentina. Profesora adjunta, Universidad Nacional del Sur, Bahía Blanca, Argentina. Dirección: San Andrés 800, Campus Universitario Altos del Palihue, 8000, Bahía Blanca, Argentina. Tel 54291 4595138. Correo electrónico: vviego@criba.edu.ar

*** Licenciada en Economía, Universidad Nacional del Sur, Bahía Blanca, Argentina. Ayudante de docencia Universidad Nacional del Sur, Bahía Blanca, Argentina. Becaria investigadora, Universidad Nacional del Sur, Bahía Blanca, Argentina. Dirección: San Andrés 800, Campus Universitario Altos del Palihue, 8000, Bahía Blanca, Argentina. Tel 54291 4595138. Correo electrónico: carolinavolonte@hotmail.com 


\section{EFFICIENCY OF URBAN PASSENGER TRANSPORTATION IN THE LOCALITY OF BAHIA BLANCA, ARGENTINA 2007-2014}

\section{ABSTRACT}

The objective of this article is to evaluate the efficiency in the urban transportation service in the city of Bahia Blanca, Argentina. The estimations where based on the stochastic border method applied to a production function with panel data for the 2007-2014 period. Results indicate that the average efficiency level didn't vary significantly during the analyzed period. At the same time, monetary transfers and line concentration allowed to maintain the business profitability without this translating necessarily in efficiency gains.

\section{KEY WORDS}

Urban mobility, passenger public transportation, stochastic method, Bahia Blanca, Argentina

\section{JEL CLASSIFICATION}

R41, R48

\section{CONTENT}

Introduction; 1. Methodology and data; 2. Results; 3. Discussion; 3. Conclusions; References.

\section{EFICIÊNCIA DO TRANSPORTE URBANO DE PASSAGEIROS NA LOCALIDADE DE BAHÍA BLANCA, ARGENTINA 2007-2014}

\section{RESUMO}

O objetivo deste artigo é avaliar a eficiência na prestação do serviço de transporte urbano por coletivo na cidade de Bahía Blanca, Argentina. As estimações se realizaram mediante a aplicação do método de fronteira estocástica a uma função de produção com dados de painel para o período 2007-2014. Os resultados indicam que o nível médio de eficiência não variou significativamente durante o período analisado. Assim mesmo, as transferências monetárias e a concentração de linhas permitiram manter a rentabilidade empresarial sem que isto se traduza em lucros de eficiência.

\section{PALAVRAS CHAVE}

Mobilidade urbana, transporte público de passageiros, fronteira estocástica, Bahía Blanca, Argentina.

\section{CLASSIFICAÇÃO JEL}

R41, R48

\section{CONTEÚDO}

Introdução; 1. Metodologia e dados; 2. Resultados; 3. Discussão; 3. Conclusões; Referências. 


\section{INTRODUCCIÓN}

Desde la década de 1990 el estudio sobre los patrones de desplazamientos de personas en el ámbito urbano, y en particular en lo referido al ordenamiento de los modos de transporte, se convirtió en uno de los más recurrentes dentro de la política territorial y las agendas políticas de países tanto desarrollados como subdesarrollados (UITP, 2009; 2007a; 2007b). En Argentina, tanto gobiernos locales como la comunidad académica comparten dicho interés, especialmente a partir del crecimiento del uso del automóvil particular y los problemas derivados (contaminación, congestión, colisiones, etc.) y la caída secular del transporte público como modalidad de desplazamiento dentro de las ciudades. Esta tendencia de largo plazo se combinó en 2002 con la crisis generada por la salida del régimen de convertibilidad de la moneda y el advenimiento posterior de períodos inflacionarios.

En el ámbito nacional, la crisis de 2002 se reflejó en una caída significativa de los volúmenes de pasajeros transportados que afectó la rentabilidad de las empresas prestatarias $^{1}$. En ese contexto la alternativa de incrementar las tarifas de transporte público no era viable ni social ni económicamente; desde el punto de vista social ello implicaba un agravamiento de la conflictividad originada en la propia crisis. Por su parte, en términos económicos el aumento de tarifas generaría inflación, en un escenario donde precisamente se trataba de contener incrementos generalizados de precios devenidos de la devaluación. Por este motivo, el Gobierno nacional puso en marcha en 2002 un sistema de subsidios a la oferta que intentaban compensar a los prestatarios del transporte denominado Sistema Integrado de Transporte Automotor (SISTAU). Durante 2002-2006 dichas transferencias fueron financiadas con impuestos al gas oil y luego con aportes del tesoro nacional. En 2006, además, la autoridad nacional de transporte puso en marcha un dispositivo adicional de reforzamiento de las compensaciones, conocido como Compensación Complementaria Provincial (CCP), cuyo monto depende de la cantidad de unidades afectadas al servicio, el combustible consumido y la plantilla de personal.

El presente trabajo centra su análisis en la ciudad de Bahía Blanca, localidad situada al sur de la provincia de Buenos Aires en Argentina y cabecera del partido homónimo. Según el último censo poblacional de 2010 residen en Bahía Blanca 301.572 habitantes.

\footnotetext{
Brevemente, esta crisis se inicia en 1998 con la caída del precio de los commodities y la reversión de la tendencia de los flujos de capitales hacia los países en desarrollo. A ello se sumaron factores internos, como la privatización del sistema de pensiones, que agravaron los resultados fiscales del Gobierno. Así, a finales de la década de 1990 la economía argentina ingresa a un período recesivo del nivel de actividad cuyo punto culminante ocurre en 2002 con la salida del régimen de convertibilidad de la moneda. Una revisión de los eventos y procesos de esta crisis puede encontrarse en Cortes (2003).
} 
Si bien habitualmente los sistemas de transporte público suele estar compuestos por varios subsistemas, en el caso particular de Bahía Blanca funcionan dos: los subsistemas de buses o colectivos y de taxis². En esta localidad, la cuestión de la movilidad urbana reviste particular interés reflejado en dos reformas en el subsistema de transporte público por colectivo que tuvieron lugar en los años 2009 y 2012.

A fines de 2007, 4 de las 7 oferentes del servicio de transporte urbano de pasajeros (que operaban 16 de las 21 líneas operativas hasta entonces) comunicaron la interrupción en la prestación del servicio bajo los argumentos de la precariedad de los contratos con el municipio (durante 40 años no fueron objeto de licitación pública), carencia de cláusulas de recomposición tarifaria y de revisión de las condiciones de operación ante variaciones en los costos de prestación del servicio. Así, en 2008 dejaron de operar 3 de las oferentes del servicio. Durante ese año se inicia el proceso de ordenamiento regulatorio del subsistema de transporte por buses, que culmina el año siguiente. La reforma de 2009 implicó la reducción de prestadores de 7 a 3 empresas privadas. En particular, 2 de los nuevos prestatarios eran controlados por un grupo empresario, lo cual significaba un nivel de concentración elevado: este prestador operaba 75 \% de las líneas de transporte público de pasajeros por colectivo3.

Luego de numerosos conflictos con los usuarios, originados en fallas en las condiciones de prestación del servicio (demoras en frecuencias, estado de las unidades, etc.), en 2012 el Gobierno local estableció la caducidad del contrato con el grupo que controlaba la mayor parte del servicio. Durante el último trimestre de 2012 se produjo una nueva organización de la oferta del sistema. La prestación continuó en manos de 3 empresas, 1 de ellas con participación mayoritaria del Gobierno municipal. Desde entonces el sistema opera 17 líneas, y su distribución entre prestadores es más homogénea que la situación previa ${ }^{4}$.

En términos generales, dichas reformas implicaron reestructuraciones en la oferta del servicio, en las que se registra la fusión de líneas, modificación de recorridos,

2 En otras ciudades, en general, ubicadas en áreas metropolitanas, suelen existir, además, subterráneo, trolebús, tranvía, tren urbano, etc.

3 El pliego de licitación establecía que ninguna empresa podía licitar más de 8 líneas. La estrategia del grupo empresario que consiguió operar el 75 \% de las líneas licitadas consistió en presentarse a la licitación a través de una firma subsidiaria.

4 Con todo, la empresa de participación estatal mayoritaria opera menos del $20 \%$ de las líneas, mientras que las privadas retienen aún más del $80 \%$ de los recorridos. 
reducción de las frecuencias, modificación de sistemas de cobro, la conformación de una empresa de participación estatal mayoritaria (SAPEM) en reemplazo de un concesionario privado, entre otras. Este proceso dio por resultado, entre otros, una mayor concentración empresarial. Dichas reformas, a su vez, se basaron en reclamos de las empresas prestatarias que argumentaban disminuciones severas de rentabilidad.

El gráfico 1 exhibe la evolución del volumen de pasajeros transportados y de los subsidios liquidados a las empresas del sector. Entre 2007 y 2009 el volumen transportado fue ascendente con un pico de 2,6 millones de pasajeros a finales de 2009. Desde 2010 la cifra de pasajeros transportados se mantiene estable (omitiendo oscilaciones cíclicas por factores estacionales y coyunturales), aunque en un nivel inferior, en torno a 2,2 millones de pasajeros por mes.

Gráfico 1. Evolución de los pasajeros totales y los subsidios en el transporte urbano colectivo en Bahía Blanca. Cifras desestacionalizadas. 2007-2014

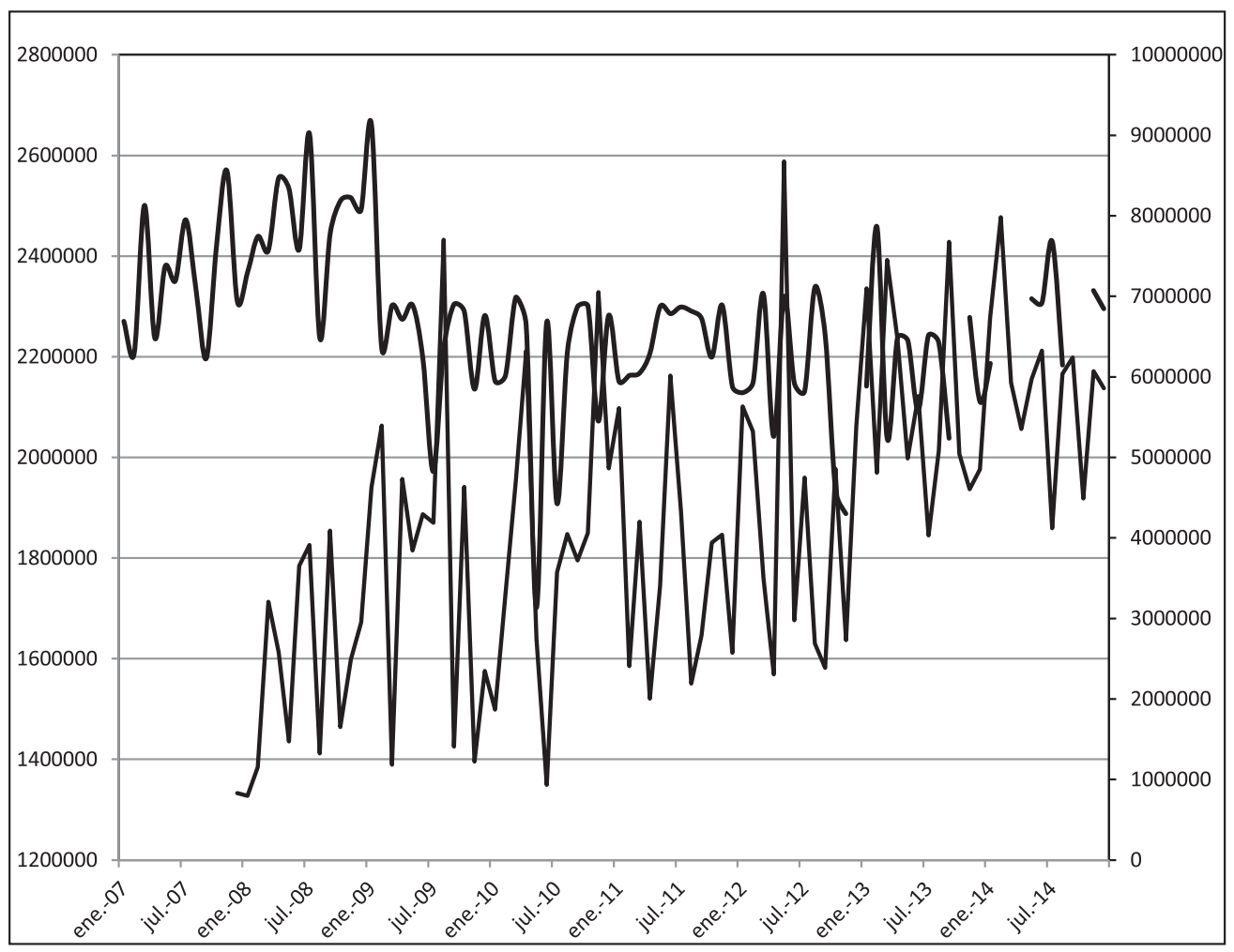

Fuente: elaboración propia basada en datos de la Secretaría de Transporte de la Municipalidad de Bahía Blanca y del Ministerio de Transporte de la Nación. 
Por su parte, la magnitud de los subsidios ha sido creciente en términos reales; mientras que el volumen de pasajeros de 2014 se ubica en cifras apenas inferiores a las de 2007, las transferencias a las empresas que prestan el servicio aumentaron 7 veces entre 2007 y 2014, aun descontando la variación de precios minoristas.

La evaluación de la eficiencia ha recibido considerable atención en la literatura de transporte público, como, por ejemplo, De Borger, Kerstenbs y Costa (2002) y Russo y Rindone (2010). En la teoría económica, la eficiencia es considerada un rasgo del proceso de producción, definido como el conjunto de actividades por medio de las cuales se combinan factores o insumos para obtener uno o más productos. A su vez, los procesos de producción se representan por medio de una función de producción que describe la relación técnica entre factores y productos. En particular, la frontera de producción es entonces la porción de la función de producción donde se alcanza el máximo producto posible con una combinación dada de insumos. Se supone que las firmas producen en niveles de frontera o por debajo de la frontera, es decir, con algún grado de ineficiencia. En otros términos, la eficiencia es la mejor relación entre recursos empleados y resultados alcanzados.

Una clasificación posible de los métodos de medición de eficiencia es el conocimiento o no de la función de producción. Por un lado, los enfoques no paramétricos se basan en el supuesto de que la función de producción de las firmas eficientes no es conocida. En este conjunto se encuentra el Análisis de Envolvente de Datos (DEA, por sus siglas en inglés) $)^{5}$. Por otro, las aproximaciones paramétricas se basan en el supuesto de que la función de producción de las empresas eficientes es conocida. El análisis de frontera estocástica (SF, por sus siglas en inglés) es el método más popular dentro de este enfoque.

Ninguno de los métodos tiene superioridad absoluta sobre el resto, de modo que la elección metodológica depende de los datos disponibles y del grado en que se cumplan los requisitos para ser aplicados ${ }^{6}$.

5 El método Free Disposal Hull (FDH, por sus siglas en inglés) es una generalización del DEA que admite, por ejemplo, indivisibilidades de insumos o productos que podrían provocar no convexidades en la tecnología y complicar la identificación de soluciones en un esquema DEA tradicional. Para una revisión de métodos no paramétricos, consultar Daraio y Simar (2007).

6 Por ejemplo, en las actividades que generan múltiples productos y ninguno predomina sobre otro, quizá se prefiera aplicar DEA antes que SF, que solo admite un producto central. 
Eficiencia del transporte urbano de pasajeros en la localidad de Bahía Blanca, Argentina 2007-2014

Tabla 1. Medición de eficiencia. Métodos

\begin{tabular}{lcc}
\hline & Enfoques no paramétricos & Enfoques paramétricos \\
\hline $\begin{array}{l}\text { Métodos } \\
\text { punción de }\end{array}$ & DEA & SF \\
\hline $\begin{array}{l}\text { Tipo de } \\
\text { eficiencia }\end{array}$ & desconocida & $\begin{array}{c}\text { conocida, con parámetros cons- } \\
\text { tantes }\end{array}$ \\
\hline $\begin{array}{l}\text { Forma de } \\
\text { medición }\end{array}$ & óptimo es considerada ineficiente & $\begin{array}{c}\text { estocástica } \\
\text { oleatorios exógenos }\end{array}$ \\
\hline
\end{tabular}

Fuente: elaboración propia basada en Brons y otros (2005)

A su vez, la eficiencia puede ser medida desde la perspectiva de producción o de costos. En particular, cuando se dispone de precios de mercado para insumos y productos, la empresa podría buscar minimizar el costo total para un nivel dado de producto.

Una revisión de los estudios empíricos que han obtenido medidas de eficiencia del transporte público urbano publicados desde el año 2005 refleja variedad en la elección del método a aplicar. Por ejemplo, la técnica DEA es empleada por García Sánchez (2009) en ciudades españolas, Carvalho, Syguiy y Silva (2015) en grandes ciudades de Brasil, Ramos, Lima y Sampaio (2008) en ciudades europeas y de Brasil, Li y otros (2013) en empresas prestatarias de transporte por colectivo en Beijing (China). Por su parte, el análisis de SF fue utilizado por Piacenza (2006) en empresas italianas de transporte por colectivo, Roy y Yvrande-Billon (2007) redes de transporte público de Francia, por Holmgren (2013) a regiones de Suecia y Jarboui, Forget y Boujelben (2015) para operadores de transporte público de 18 países ${ }^{7}$. En particular, la mayoría de estudios empíricos revisados que emplean SF estiman fronteras de costo.

La valoración de ambos enfoques de Brons y otros (2005) sugiere que los métodos paramétricos pueden producir mayores valores de eficiencia que los no paramétricos, ya que una parte de la brecha entre el producto efectivo y el óptimo es atribuida a errores de medición y shocks aleatorios, mientras que en los enfoques determinísticos toda diferencia es ineficiencia. Sin embargo, el metaanálisis que realizan sugiere que, cuando se emplean unidades físicas del nivel de producción, las medidas de eficiencia son menores que cuando se utilizan variables monetarias o vinculadas a recaudación.

$\overline{7} \quad$ El listado de referencias se engrosa, si el horizonte de revisión se amplía. 
El objetivo del trabajo es estimar una función de producción del servicio público de transporte por colectivo para la ciudad de Bahía Blanca. La estimación permite: a) identificar la sensibilidad de la producción de cada empresa prestataria del servicio al volumen de unidades afectadas al servicio, a la extensión del recorrido y a la cantidad de líneas que opera, y b) evaluar la evolución de la eficiencia técnica del sector. Conviene aclarar que, al tratarse de empresas de servicios, su nivel de producción se mide por el volumen de pasajeros transportados.

El artículo se organiza del siguiente modo: en la sección 1 se presenta la metodología a emplear y los datos disponibles para realizar las estimaciones. La segunda sección expone los resultados obtenidos, puntualiza los parámetros significativos y la interpretación de otros indicadores de interés. La sección 3 propone una discusión del alcance y limitaciones del trabajo. Finalmente, en la sección 4 se resumen las ideas centrales.

\section{METODOLOGÍA Y DATOS}

La metodología empleada se basa en la estimación de una función de producción de transporte de pasajeros con método de frontera estocástica. SF supone que el nivel de producción de una determinada empresa no se determina unívocamente a partir del nivel de insumos y factores empleados, sino que puede estar influido por elementos fuera de su control, aleatorios, capturados en el término de perturbación. En ese sentido, el nivel de producción observado puede diferir del nivel óptimo, tanto por defectos en la gestión de la empresa como por contingencias no observables.

La estimación de funciones de producción mediante el método de frontera estocástica descompone entonces el término de perturbación en dos tipos: por un lado, el componente puramente aleatorio (que puede reducir o incrementar los niveles de producción óptimos) y, por otro, la ineficiencia de la empresa (que siempre reduce la producción respecto del óptimo).

Esta descomposición requiere suponer una forma funcional específica para la función de producción y una distribución para el término de perturbación. Es por ello que se trata de un enfoque paramétrico ${ }^{8}$. La función de producción habitual en este enfoque es de tipo Cobb-Douglas y toma la siguiente especificación:

\footnotetext{
${ }^{8}$ Existen otras formas de obtener indicadores de eficiencia de las empresas que son independientes de la forma funcional adoptada. Se trata de métodos no econométricos, que se resuelven con técnicas de programación lineal. Por ello, se conocen como no paramétricos y el enfoque más popular es el conocido como Envolvente de Datos (DEA, por sus siglas en ingles). Una comparación de ambos enfoques se encuentra en Fried, Lovell y Schmidt (2007).
} 


$$
\ln \mathrm{O}_{i t}=\beta_{0}+\sum_{k=1}^{k} \beta_{k} \ln \mathrm{X}_{\text {kit }}+v_{i t}-u_{i t} \operatorname{con} i: 1, \ldots, \mathrm{N} \mathrm{y} t: 1, \ldots, \mathrm{T}
$$

Donde $\mathrm{Q}_{i t}$ es el nivel de producción de la empresa $i$ perteneciente al sector del transporte urbano de pasajeros, medida por el total anual de pasajeros que utilizan las líneas pertenecientes a la empresa $i, X_{k}$ es una matriz de kxk que reúne las variables explicativas consideradas. En este caso particular, se incluyen el parque móvil (PAR), kilómetros recorridos (KIM), $\beta$ es el vector de los parámetros a estimar y corresponde a la elasticidad del producto de nivel frontera respecto a cada insumo considerado. En este caso, al considerar 2 explicativas $\beta$ tiene 2 elementos. El subíndice $i$ representa a cada una de las empresas que conforman el sector, donde $\mathrm{N}$ es igual a 10 y $t$ simboliza el período, en este caso, expresado con frecuencia anual, siendo $\mathrm{T}=8$ años? .

Por último, la expresión $v_{i t-} u_{i t}$ representa el error compuesto antes mencionado, donde $v_{i t}$ simboliza los shocks puramente aleatorios y externos a la empresa, se supone idénticamente distribuido, siguiendo una distribución normal, $N\left(0, \sigma^{2}\right)$, $\mathrm{y}$ es independiente del segundo componente del error, $u_{i}$, que representa el nivel de ineficiencia de la empresa i en el período t. En este caso, el modelo de frontera estocástica se estima imponiendo la restricción $u_{i t} \geq 0$, en tanto se supone que la empresa se encuentra en la frontera (es decir, con $100 \%$ de eficiencia) o por debajo de ella (con ineficiencia).

Con respecto a $u_{i t}$, se consideraron dos parametrizaciones posibles: puede suponerse invariante en el tiempo o, por el contrario, variante en $t$. La especificación de esta última opción propuesta por Battese y Coelli (1992) es:

$$
u_{i t}=e^{-\eta\left(t-T_{i}\right) u_{i}}
$$

donde $\mathrm{T}_{\mathrm{i}}$ representa el último período de la i-ésima empresa. En particular, cuando $t=T_{i}$, la ineficiencia obtenida es el nivel de base de la ineficiencia de la empresa $i$. Por su parte, $\eta$ es el parámetro de descenso e informa sobre la dirección que toma la ineficiencia en el tiempo; cuando $\eta>0$, el nivel de ineficiencia decrece hasta el nivel base las empresas se desplazan hacia la frontera a una tasa de (100 x $\eta) \%$ por año. Inversamente, cuando $\eta<0$, la ineficiencia técnica aumenta exponencialmente, lo cual implica divergencia tecnológica.

Tanto en la opción de eficiencia estática como cambiante en el tiempo, $u_{i t}$ sigue una distribución normal-truncada en $0, u_{i t} \sim \mathrm{N}^{t}\left(\mu, \sigma_{u}^{2}\right)$.

\footnotetext{
9 T representa el último período del panel, que no necesariamente será igual para todas las empresas, tanto porque hay datos faltantes de algunos períodos como porque las empresas funcionaron en distintos períodos de tiempo, de acuerdo con las reformas regulatorias.
} 
En este caso, se espera que el modelo más adecuado sea el que admite variaciones en la ineficiencia en el tiempo, a la luz de los cambios regulatorios ocurridos. En particular, se esperaría que el ingreso de una empresa con gestión estatal y la desconcentración de la oferta generasen una reducción de la ineficiencia, al menos de la observada durante el período de elevada concentración empresarial previo (2009-2012).

Por otro lado, si bien la mayor parte de estudios empíricos que miden eficiencia del transporte urbano estima fronteras de costo, en este caso se estiman fronteras de producción por carencia de datos de costo desagregados por firma.

Ambas variantes sobre la forma evolutiva de $u_{i t}$ fueron estimadas aplicando el método de máxima verosimilitud. Las estimaciones fueron realizadas con el paquete econométrico Stata v 12.

Las variables explicativas escogidas son similares a las empleadas por Pestana y otros $(2008)^{10}$.

Como subproducto de la estimación, se analizó el efecto de la concentración de líneas por empresa y de los subsidios al transporte urbano sobre el nivel estimado de ineficiencia, $u_{i t}$. Este segundo modelo fue estimado con datos de panel y efectos fijos, de modo de considerar las especificidades de cada empresa, en particular, la ecuación estimada sobre los factores que influyen sobre la eficiencia de los prestadores de transporte urbano sigue la forma:

$u_{i t}=\alpha_{0 i}+\alpha_{1} \operatorname{LIN}_{i t}+\alpha_{2} \mathrm{CCP}_{i t}+\alpha_{3} \operatorname{SISTAU}_{i t}+v_{i t}$

donde LIN representa la cantidad de líneas que la i-ésima empresa opera en el período t, CCP y SISTAU registran los montos (en moneda constante) de las transferencias del Ministerio de Transporte a las empresas prestatarias del servicio de transporte urbano en compensación por incremento de costos.

Los datos empleados ${ }^{11}$ y las fuentes de información se detallan en el tabla 2.

10 Estos últimos, sin embargo, estiman una función de frontera estocástica de costo para el transporte urbano de pasajeros de Francia en su conjunto.

11 El lector que desee acceder a la base de datos utilizada en las regresiones puede solicitarlo al siguiente correo: vviego@criba.edu.ar. 
Eficiencia del transporte urbano de pasajeros en la localidad de Bahía Blanca, Argentina 2007-2014

Tabla 2. Definición de variables empleadas y fuentes de información

\begin{tabular}{|c|c|c|}
\hline Variable & Definición & Fuente de información \\
\hline \multicolumn{3}{|c|}{ Dependiente } \\
\hline Q & pasajeros totales anuales & $\begin{array}{l}\text { Dirección de Tránsito y Transporte de la } \\
\text { Municipalidad de Bahía Blanca }\end{array}$ \\
\hline \multicolumn{3}{|c|}{ Explicativas del nivel de producción del servicio } \\
\hline KIM & $\begin{array}{l}\mathrm{km} \text { anuales recorridos. Suma de km } \\
\text { mensuales transitados }\end{array}$ & $\begin{array}{l}\text { Comisión Nacional de Regulación del } \\
\text { Transporte }\end{array}$ \\
\hline PAR & $\begin{array}{l}\text { número de unidades afectadas al } \\
\text { servicio }\end{array}$ & $\begin{array}{l}\text { Comisión Nacional de Regulación del } \\
\text { Transporte }\end{array}$ \\
\hline \multicolumn{3}{|c|}{ Explicativas del nivel de eficiencia } \\
\hline LIN & número de líneas operadas & $\begin{array}{l}\text { Sitio web de la empresa Rastreador Fournier } \\
\text { (http://www.rastreadorfournier.com.ar/) } \\
\text { Notas periodísticas }\end{array}$ \\
\hline ССР & $\begin{array}{l}\text { transferencias recibidas por com- } \\
\text { pensación de mayores costos com- } \\
\text { bustible, en pesos, deflactadas por } \\
\text { IPC }(2007 \mathrm{ml} 1=100)^{*}\end{array}$ & $\begin{array}{l}\text { Ministerio de Transporte de la Nación. http:// } \\
\text { www.transporte.gob.ar/content/subsidios/ } \\
\text { IPC State Street, Price Stats. www.states- } \\
\text { treet.com }\end{array}$ \\
\hline SISTAU & $\begin{array}{l}\text { transferencias recibidas por com- } \\
\text { pensación de mayores costos globa- } \\
\text { les, deflactadas por IPC State Street } \\
(2007 \mathrm{~m} 11=100)\end{array}$ & $\begin{array}{l}\text { Ministerio de Transporte de la Nación. http:// } \\
\text { www.transporte.gob.ar/content/subsidios/ } \\
\text { IPC State Street, Price Stats www.statestreet. } \\
\text { com }\end{array}$ \\
\hline
\end{tabular}

* Se utiliza el IPC Congreso, alternativo al índice de precios oficial, debido al cuestionamiento que este último indicador ha recibido tanto de ámbitos académicos como políticos o periodísticos por su severa subestimación de la variación de los precios minoristas en Argentina. El índice empleado es difundido mensualmente por una comisión de la Cámara de Diputados y se calcula como el promedio de 9 índices elaborados por institutos provinciales de estadística y consultoras privadas.

Fuente: Cálculos propios

\section{RESULTADOS}

Al analizar los resultados arrojados para los parámetros $\beta_{k}$ estimados (tabla 3), en ambos modelos se observó que tanto el parque móvil como los kilómetros recorridos resultaron significativos. Sin embargo, el signo que acompaña al coeficiente vinculado al parque móvil es contrario al esperado. Esto se debe a la elevada multicolinealidad entre los regresores empleados ${ }^{12}$, que genera el riesgo de anular la significancia individual de los coeficientes o, como en este caso, invertir su signo ${ }^{13}$.

12 El coeficiente de correlación simple entre ambas es superior a 0.9

13 Los coeficientes estimados son superiores en magnitud a los obtenidos por Pestana et al. (2008), aunque estrictamente no serían comparables, ya que en este caso se estima una función de producción, mientras que el trabajo citado estima funciones de costo. 
El parámetro que refleja la evolución del término de ineficiencia, $\eta$, no resultó significativo al $1 \%$, lo que indicaría que la ineficiencia técnica del conjunto de prestatarios del servicio de transporte de pasajeros en Bahía Blanca durante 2007-2014 se mantuvo sin cambios apreciables. En estos términos, los cambios regulatorios no habrían conseguido, al menos hasta 2014, un incremento en la eficiencia.

Por su parte, el valor del parámetro $\gamma$, que expresa el porcentaje de la varianza total explicada por la varianza de la $u_{i t}$, indica que casi $87 \%$ de la varianza del término de perturbación compuesto proviene de la varianza del término de ineficiencia.

Con respecto a $\mu$, que representa la estimación del valor medio de la distribución normal truncada, resultó significativo al $5 \%$, lo que equivale a afirmar que la ineficiencia promedio es estadísticamente distinta de 0.

Tabla 3. Transporte público de pasajeros de Bahía Blanca. Estimaciones de frontera estocástica 2007-2014

\begin{tabular}{ll}
\hline & \multicolumn{1}{c}{$\begin{array}{c}\ln \mathrm{Q}, \\
\text { pasajeros totales }\end{array}$} \\
\hline Constante, $\beta_{0}$ & $\begin{array}{l}2,872^{* *} \\
(1,171)\end{array}$ \\
\hline $\ln$ PAR, $\beta_{1}$ & $\begin{array}{l}-0,513^{* *} \\
(0,256)\end{array}$ \\
\hline $\ln \mathrm{KIM}, \beta_{2}$ & $1,104^{* * *}$ \\
\hline Mu, $\mu$ & $(0,082)$ \\
\hline Eta, $\eta$ & $1,725^{* *}$ \\
\hline$\sigma^{2}$ & $(0,81)$ \\
\hline$\gamma$ & 0,005 \\
& $(0,019)$ \\
\hline No. observaciones & 0,395 \\
\hline
\end{tabular}

*** Significativas al $1 \% .{ }^{* *}$ significativas al $5 \% .{ }^{*}$ significativas al $10 \%$.

Fuente: Cálculos propios

Un análisis de la evolución de la eficiencia promedio del sistema en su conjunto en el período analizado muestra la ausencia de una tendencia definida; entre 2007 y 2009 la eficiencia del sistema aumentó, unos 4 puntos porcentuales; luego, en los años siguientes a la reforma de 2009, se registra una disminución de la eficiencia y desde 2011 la eficiencia aumentó, aunque a niveles inferiores al pico de 2009. Con 
todo, estas variaciones no son suficientes para reflejar cambios estadísticamente significativos. Por otra parte, se deben resaltar los valores considerablemente bajos de eficiencia, que indican niveles de ineficiencia superiores al $70 \%{ }^{14}$.

Gráfico 2. Evolución de la eficiencia promedio del sistema de transporte por colectivo. Bahía Blanca. 2007-2014

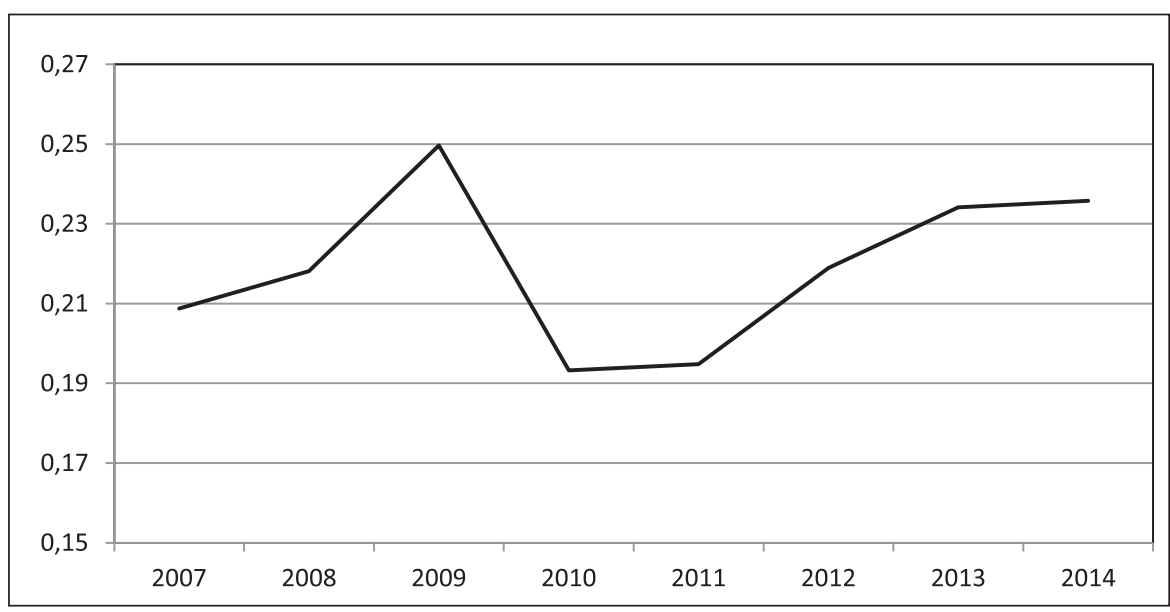

Fuente: elaboración propia

El gráfico 3 presenta las estimaciones de eficiencia promedio por empresa con indicación de los períodos en que operó cada una. Los valores más elevados corresponden a empresas que prestan el servicio desde la última reforma, con valores cercanos o superiores al $20 \%$ mientras que las prestatarias que ofrecieron el servicio entre 2007-2009 exhiben niveles de eficiencia promedio menores, a excepción de una empresa que operó durante 2007 y los primeros meses de 2009, que registra niveles de eficiencia superiores al $70 \%$.

Por su parte, la estimación de la ecuación 3 indica un efecto contrapuesto de las transferencias que, en términos netos, sería desfavorable ${ }^{15}$; mientras que los subsidios al combustible reducirían las ineficiencias, las transferencias del sistema SISTAU incrementarían la ineficiencia. Por ende, las transferencias por compensaciones de costos se han traducido en un menor nivel de pasajeros respecto del óptimo.

14 Vale la pena recordar que, para facilitar la interpretación, el análisis de (in)eficiencia se presenta en términos porcentuales, donde la eficiencia es el ratio entre el nivel de servicio observado y aquel estimado como óptimo.

15 El test que chequea si $\alpha 2+\alpha 3=0$ arroja un estadístico $F=7,4$ con un $p$-valor $=0,0215$, lo cual implica que al $5 \%$ hay evidencia de que el efecto neto sobre la ineficiencia sería positivo y, por ende, perjudicial. 
Dicho de otro modo, los subsidios al transporte deberían haberse traducido en un volumen mayor de tráfico de pasajeros.

Gráfico 3. Eficiencia promedio por empresa. Bahía Blanca. 2007-2014

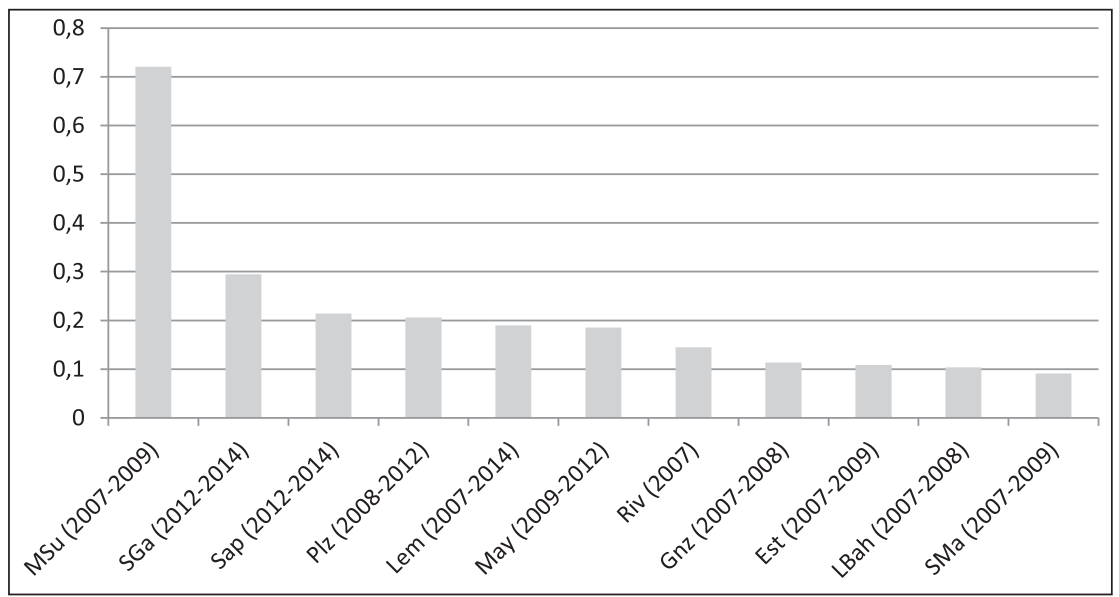

Fuente: cálculos propios

Tabla 4. Determinantes de la ineficiencia en el transporte por colectivos. Bahía Blanca. 2007-2014

\begin{tabular}{lc}
\hline LIN & $u_{i t}$ \\
\hline CCP & $\begin{array}{c}-0,0001^{*} \\
(0,0001)\end{array}$ \\
\hline SISTAU & $\begin{array}{c}-1,15 \times 10^{-9 * * *} \\
\left(2,37 \times 10^{-10}\right)\end{array}$ \\
\hline$R^{2}$ dentro & $\begin{array}{c}2,94 \times 10^{-9 * * *} \\
\left(7,64 \times 10^{-10}\right)\end{array}$ \\
\hline$R^{2}$ entre & 0,7657 \\
\hline$R^{2}$ global & 0,0011 \\
\hline $\mathrm{F}(2,23)$ & 0,0005 \\
\hline
\end{tabular}

Errores estándar robustos a la heterocedasticidad

*** Significativas al $1 \%$. ${ }^{* *}$ significativas al $5 \%$. ${ }^{*}$ significativas al $10 \%$.

Fuente: Cálculos propios

Este resultado no implica la recomendación de eliminar los subsidios y permitir aumentos tarifarios que, probablemente, también reducirían la brecha entre pasajeros transportados y el óptimo, esta vez, por reducción de la demanda. 
Por su parte, la evidencia de que la concentración de líneas favorecería la eficiencia es débil.

Lo que reflejan ambos resultados (efectos de los subsidios y de la concentración de líneas) es que las transferencias monetarias y la posibilidad de operar varias líneas permiten mantener la rentabilidad empresarial, sin que ello se traduzca en forma directa en ganancias de eficiencia. Este factor depende, en buena medida, de la capacidad de control del regulador y de su compromiso con los niveles y calidad de prestación del servicio.

\section{DISCUSIÓN}

Los resultados anteriores deben ser evaluados a la luz de ciertas consideraciones en torno a las economías de escala y al vínculo entre eficiencia y calidad.

Farsi, Filippni y Kuenzle (2006) discuten la forma de evaluar la existencia de economías de escala en el transporte urbano de pasajeros y consideran que la extensión de la red (que puede aproximarse, por ejemplo, por la cantidad de líneas que opera cada empresa) es un indicador de economías de escala. En particular, las estimaciones mencionadas antes de Farsi, Filippni y Kuenzle (2006) para fronteras de costo arrojan la existencia de economías de escala en el sector de transporte urbano de pasajeros. En otros términos, la operación de una red más extensa permitiría disminuir más que proporcionalmente los costos medios. Los resultados encontrados para Bahía Blanca indicarían que la cantidad de líneas disminuye la ineficiencia, aunque su significancia tanto estadística como económica es débil, sugiriendo la ausencia de economías de escala significativas basadas en la extensión de la red, en coincidencia con lo señalado por Nieswand, Hess y Von Hirschhausen (2008) para compañías de transporte de tamaño pequeño y mediano (en términos de la flota en operación). De todos modos, este resultado requeriría un período más extenso de tiempo y de operadores con el fin de obtener mayor variabilidad en las variables involucradas que permita valorar la robustez de este resultado.

Otro punto central en el análisis de eficiencia que no pudo ser abordado aquí por carencia de información confiable es el referido a la existencia de dilemas (trade-off) entre eficiencia y calidad del servicio. Por ejemplo, el aumento en el volumen de pasajeros transportados respecto del óptimo podría hacerse sacrificando frecuencias (con el consecuente aumento del tiempo de espera en las paradas con el fin de reunir mayor cantidad de pasajeros que no pueden desplazarse en otro medio de transporte), o a expensas del confort en el viaje (admitiendo mayor cantidad de pasajeros que de asientos). 
Un análisis de las estimaciones de eficiencia obtenidas junto con artículos periodísticos publicados en la prensa daría cuenta de que los aumentos de eficiencia de 2007-2009 ocurrieron a expensas de frecuencias y confort, mientras que entre 2010-2011 disminuyó tanto la eficiencia como la calidad del servicio. No se dispone de información suficiente para especular sobre la relación eficiencia-calidad desde 2012 en adelante.

\section{CONCLUSIONES}

Con este trabajo se pretende contribuir al análisis de la eficiencia de los operadores del transporte público de pasajeros en la ciudad de Bahía Blanca a partir de una base de datos con información correspondiente a 11 empresas que sucesivamente prestaron el servicio entre 2007 y 2014. Mediante la aplicación de la metodología de frontera estocástica se estimó una función de producción del tipo Cobb-Douglas que incluye las unidades en operación, las distancias recorridas y la cantidad de líneas adjudicadas a cada empresa.

Entre los resultados significativos se encuentra que el nivel de eficiencia no se ha modificado significativamente desde 2007, aún a pesar de la salida de operadores con prestaciones deficientes del servicio (los porcentajes de eficiencia han oscilado entre $19 \%$ y $25 \%$, aunque las pruebas estadísticas indican que su variación no ha sido significativa). Por su parte, el incremento en términos reales de los subsidios otorgados por el Gobierno nacional no se tradujo en mayores niveles de eficiencia.

\section{BIBLIOGRAFÍA}

Battese, G. y Coelli, T. (1992). Frontier production functions, technical efficiency and panel data: With application to paddy farmers in India. En: Journal of Productivity Analysis Vol. 3, No. 1/2, Special Issue: International Applications of Productivity and Efficiency Analysis, p. 153-169.

Brons M.; Nijkamp P.; Pels E. y Rietveld P. (2005). Efficiency of urban public transit: A meta analysis. En: Transportation Vol. 32, No. 1, p. 1-21.

Carvalho M, Syguiy T, y Silva D. (2015). Efficiency and Effectiveness Analysis of Public Transport of Brazilian Cities. En: Journal of Transport Literature, Vol 9, No. 3, p. 40-44.

Cortes Conde, R. (2003); La crisis argentina de 2001-2002. En: Cuadernos de Economía, Vol. 40, No. 121, p.762-767.

Daraio, C y Simar, L. (2007); Advanced robust and non parametric methods in efficiency analysis; Springer. 248p.

De Borger, B., Kerstens, K. y Costa, A. (2002). Public transit performance: What does one learn from frontier studies? En: Transport Reviews, Vol. 22, No. 1, p. 1 38. 
Farsi M.; Filippni M. y Kuenzle M. (2006). Cost efficiency in regional bus companies: an application of alternative stochastic frontier models. En: J Transport Econ and Pol; Vol. 40, No. 1, p. 95-118.

Fried, H.; Lovell, K. y Schmidt, S. (2007). The measurement of productive efficiency and productivity. Oxford University Press, 656p.

Garcia Sanchez, I. M. (2009). Technical and scale efficiency in Spanish urban transport: Estimating with Data Envelopment Analysis. En: Advances in Operations Research, Vol 2009, Article ID 721279, 15p. Doi.org/10.1155/2009/721279.

Holmgren, J. (2013). The efficiency of public transport operations: An evaluation using stochastic frontier analysis. En: Research in Transportation Economics, Vol. 39, No. 1, p. 50-57.

Jarboui S; Forget, P. y Boujelben, Y. (2015). Efficiency evaluation in public road transport: a stochastic frontier analysis. En: Transport, Vol. 30, No. 1, p. 1 14. Doi: 10.3846/16484142.2013.785019.

Li, J.; Chen, X.; Li, X. y Guo, X. (2013); Evaluation of Public Transportation Operation based on Data Envelopment Analysis. En: Procedia - Social and Behavioral Sciences, Vol. 96, No. 6, p. $148-155$.

Nieswand, M.; Hess, B. y von Hirschhausen, Ch. (2008); Cost efficiency and market structure in German public bus transport; Deutsches Institut für Wirtschaftsforschung, WP EA 09, 23p.

Pestana Barros, C.; Guironnet, J-P.; Peypoch, N. y Roy, W. (2008). Heterogeneity in Technical Efficiency of the French Urban Transport: 1995 to 2002. School of Economics and Management Technical University of Lisbon, Departament of Economics, WP 17/2008/DE/UECE, 25p.

Piacenza, M. (2006). Regulatory Contracts and Cost Efficiency: Stochastic Frontier Evidence from the Italian Local Public Transport. En: Journal of Productivity Analysis, Vol. 25, No. 3, p. 257-277.

Russo, F. y Rindone, C. (2010); Evaluation methods for evacuation planning, p. 335-344. En: A. Pratelli y C. Brebbia (Eds.). Urban Transport XVI; WIT Press, 3687p.

Ramos Sampaio, B.; Lima Neto, O. y Sampaio, Y. (2008); Efficiency analysis of public transport systems: Lessons for institutional planning. En: Transportation Research Part A: Policy and Practice, Vol. 42, No. 3, p. 445-454. Doi:10.1016/j.tra.2008.01.006.

Roy, W. y Yvrande-Billon, A. (2007). Ownership, Contractual Practices and Technical Efficiency: The Case of Urban Public Transport in France. En: Journal of Transport Economics and Policy, Vol. 41, No. 2, p. 257-282.

UITP -Union Internationale des Transports Publiques- (2009). Integración del transporte público y de la planificación urbana: por un círculo virtuoso. Enero, 6p.

UITP -Union Internationale des Transports Publiques- (2007a). Urban mobility and congestion charging. Junio, $4 \mathrm{p}$.

UITP -Union Internationale des Transports Publiques- (2007b). Tackling social exclusion. The role of public transport. Mayo, $4 \mathrm{p}$. 
